\title{
Comparison of Two Real-Time Predictive Strategies for the Optimal Energy Management of a Hybrid Electric Vehicle
}

\author{
R. Beck, A. Bollig and D. Abel \\ Institute of Automatic Control, RWTH Aachen University, Steinbachstrasse 54, 52074 Aachen - Germany \\ e-mail: r.beck@irt.rwth-aachen.de
}

Résumé - Comparaison de deux stratégies temps réel prédictives pour la gestion d'énergie optimale d'un véhicule électrique hybride - La présence d'au moins deux sources de puissance alternatives dans un véhicule électrique hybride pose le problème de la détermination du partage de puissance entre celles-ci. Le partage optimal tient compte de la demande du conducteur et mène à une consommation de carburant minimale. Cet article présente deux stratégies qui donnent une solution à ce problème d'optimisation en utilisant des informations prédictives concernant les conditions de la voie dans un horizon temporel futur limité. Les aspects importants en ce qui concerne l'optimalité du partage de puissance déterminé et l'applicabilité en temps réel des algorithmes sont aussi abordés.

\begin{abstract}
Comparison of Two Real-Time Predictive Strategies for the Optimal Energy Management of a Hybrid Electric Vehicle - The presence of at least two alternative power sources in a HEV poses the problem of determining the optimal power split among them for a given driver's request in order to achieve a minimum fuel consumption. This article will introduce two strategies capable of solving this optimisation problem both relying on predictive information about the driving conditions within a limited future time horizon. The important aspects of optimality of the determined power split and the real-time applicability of the algorithms are addressed.
\end{abstract}




\section{INTRODUCTION}

Rising fuel prices and the tightened emission legislation have led to an increasing effort in improving the fuel efficiency of automotive vehicles. One possible solution which has drawn much attention during the last few years is the hybridisation of the conventional powertrain. Adding an appropriate second source of power to the Internal Combustion Engine (ICE) together with an energy storage device adds an additional degree of freedom to the operation strategy of the vehicle since the propulsion energy can be delivered by the single traction unit or the combination of them most appropriate for the current driving conditions. The hybridisation solution currently favoured is the combination of an ICE, one or possibly more electric machines (EM) and a battery of high capacity leading to a Hybrid Electric Vehicle (HEV).

The determination of the optimal power split among the ICE and the electric machines is the key issue for achieving a high fuel economy. The optimality can thereby be quantified as the minimum fuel consumption over the current drive cycle observing a charge sustaining operation of the vehicle whereby in general neither the duration nor the exact load profile of the drive cycle are known in advance. This article will assume that, as current research focusses on appropriate sensor technology and telematics, the vehicle's velocity and the road slope can be predicted over a limited future time segment. This consequently leads to a receding horizon control problem which consists of finding the optimal power split trajectory that minimises the fuel consumption for the predicted drive conditions. Thereby only the current optimal power split is applied and the calculations are repeated in the next time step using updated predictions and system states. The resulting constrained nonlinear optimisation problem in each time step is difficult to solve in real-time. Several solutions have been proposed in the literature among them the development of customised dynamic programming schemes [1,2] and the application of a local optimisation strategy based on the concept of weighting the consumption of fuel energy against the use of electrical energy by means of an equivalence factor [3-5]. The latter concept converts the constraint of charge sustainment over the drive cycle into the problem of a correctly chosen equivalence factor.

The work presented in this paper focuses on the aspect of real-time applicability of an optimal energy management strategy. Despite the customised numerical schemes and problem reformulations which led to significant improvements in terms of computing time, previous solutions still suffer from the nonlinearity of the optimisation problem that has to be solved iteratively. This paper shows that, rather than focusing on improved numerical algorithms, a suitable approximation of the minimisation problem and the underlying vehicle model enables an explicit solution with a significantly reduced computational burden. In a first step it is demonstrated how the well-known Adaptive Equivalence concept benefits from this system approximation due to the efficient explicit calculation of the charge sustaining equivalence factor. In a second step it is shown how the original nonlinear optimisation problem, which up to now has been solved by time-consuming dynamic programming techniques, reduces to a mixed integer quadratically constrained linear program which enables a fast direct solution. A comparison exhibits the superiority of the latter approach in terms of optimality.

\section{SYSTEM MODELLING}

The proposed optimal energy management strategies are examined exemplarily for (but are not limited to) the rearwheel driven parallel hybrid drivetrain structure displayed in Figure 1. In order to provide the capability of pure electrical propulsion, the ICE can be disconnected from the drivetrain by a clutch.

\subsection{Nonlinear Quasistatic Model}

The system model has been developed according to the wellknown quasistatic approach [6]. Thereby the dynamics of the system are inverted and the torque required at the wheels $T_{w h}$ and the angular velocity of the rear axle $\omega_{w h}$ can be calculated as

$$
\begin{aligned}
\omega_{w h}= & \frac{v}{r_{w h}(v)} \\
T_{w h}= & r_{w h}(v)\left(\frac{\rho}{2} A_{f} c_{d} v^{2}+f_{r}(v) m g \cos \alpha+m g \sin \alpha\right) \\
& +\frac{J_{t o t}}{r_{w h}(v)} \frac{d v}{d t}
\end{aligned}
$$

for a given vehicle speed $v$ and a known road slope $\alpha$. The vehicle parameters are the frontal area $A_{f}$, the drag coefficient $c_{d}$, the rolling resistance coefficient $f_{r}$, the vehicle mass $m$, the wheel radius $r_{w h}$ and the total vehicle inertia $J_{t o t}$. The angular velocity $\omega_{\text {in }}$ and the torque request $T_{\text {in }}$ at the gearbox input are then given by

$$
\begin{aligned}
& \omega_{\text {in }}= \omega_{w h} i_{0}(g) \\
& T_{i n}= \begin{cases}\frac{T_{w h}+T_{\text {loss }}\left(\omega_{w h}, g\right)}{i_{0}(g) \eta(g)}, & T_{w h}+T_{\text {loss }} \geq 0 \\
\frac{T_{w h}+T_{\text {loss }}\left(\omega_{w h}, g\right)}{i_{0}(g)} \eta(g), T_{w h}+T_{\text {loss }}<0\end{cases}
\end{aligned}
$$

where $T_{\text {loss }}$ denominates additional losses caused by friction, $i_{0}$ the total transmission ratio and $\eta$ the total transmission efficiency from the gearbox input to the rear wheels. The corresponding gear $g$ can be calculated using the specified shift schedules of the automatic gearbox. 


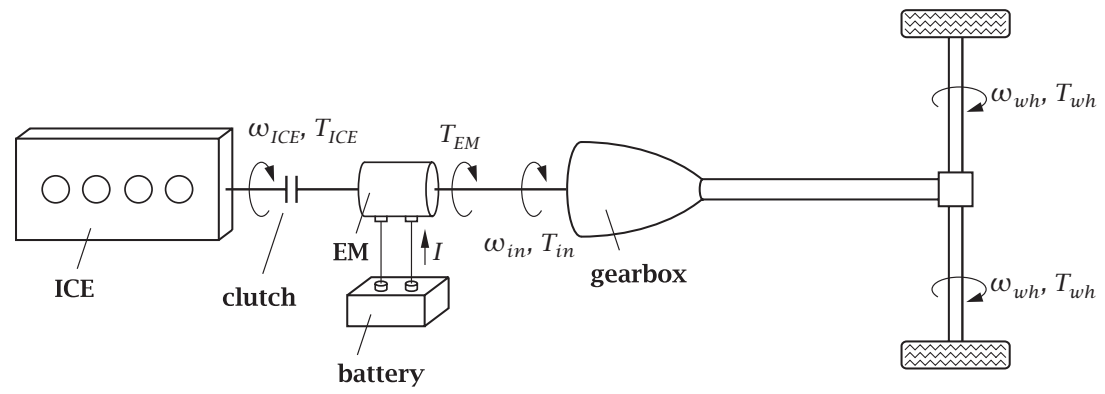

Figure 1

System architecture.

The torque request $T_{\text {in }}$ has to be satisfied by the ICE and the EM yielding

$$
T_{\text {in }}=T_{E M}+T_{I C E}
$$

The fuel consumption of the engine

$$
\dot{m}_{f}=f_{m f}\left(T_{I C E}, \omega_{I C E}\right)
$$

is given by the engine map where $\omega_{I C E}$ is equal to $\omega_{i n}$ if the clutch is closed and zero otherwise. The electrical power delivered or consumed by EM

$$
P_{e l}=f_{P e l}\left(T_{E M}, \omega_{i n}\right)
$$

can also be extracted from a corresponding map. The battery is modelled as a voltage source with an open circuit voltage $U_{0}(\xi)$ and an inner resistance $R_{i}(\xi)$ both depending on the state of charge (SOC) $\xi$ of the battery. This leads to the current

$$
I=\frac{U_{0}(\xi)-\sqrt{U_{0}(\xi)^{2}-4 P_{e l} R_{i}(\xi)}}{2 R_{i}(\xi)}
$$

of the electric circuit. The evolution of the state of charge is given by

$$
\dot{\xi}=-\frac{I}{Q_{\max }}
$$

with the maximum capacity $Q_{\max }$ of the battery.

\subsection{Approximate Quasistatic Model}

Equations (1)-(9) yield a nonlinear dynamic system (with only one state $\xi$ ) which leads to a computationally demanding nonlinear dynamic program if the fuel optimal split between $T_{I C E}$ and $T_{E M}$ is to be found [1,2]. The key point in reducing this computational complexity in order to ensure real-time capability is to approximate the nonlinear model in an appropriate way that allows the application of fast and efficient optimisation schemes, which will be demonstrated in the following.
It is known that the fuel consumption increases almost linearly with an increasing torque request at a fixed angular velocity $\omega_{I C E}$ yielding the approximation

$$
f_{m f}\left(T_{I C E}, \omega_{I C E}\right) \approx a_{0}\left(\omega_{I C E}\right) s_{I C E}+a_{1}\left(\omega_{I C E}\right) T_{I C E}
$$

with

$$
s_{\text {ICE }}= \begin{cases}1, & \text { ICE on } \\ 0, & \text { ICE off }\end{cases}
$$

Figure 2 depicts this behaviour exemplarily for the engine used for the simulations described in this article. Since $a_{0} \neq 0$, this approximation captures the main feature of a decreasing specific fuel consumption with an increasing engine torque.

Since the battery is usually operated within tight SOC limits due to durability considerations, the effect of $\xi$ in (8)

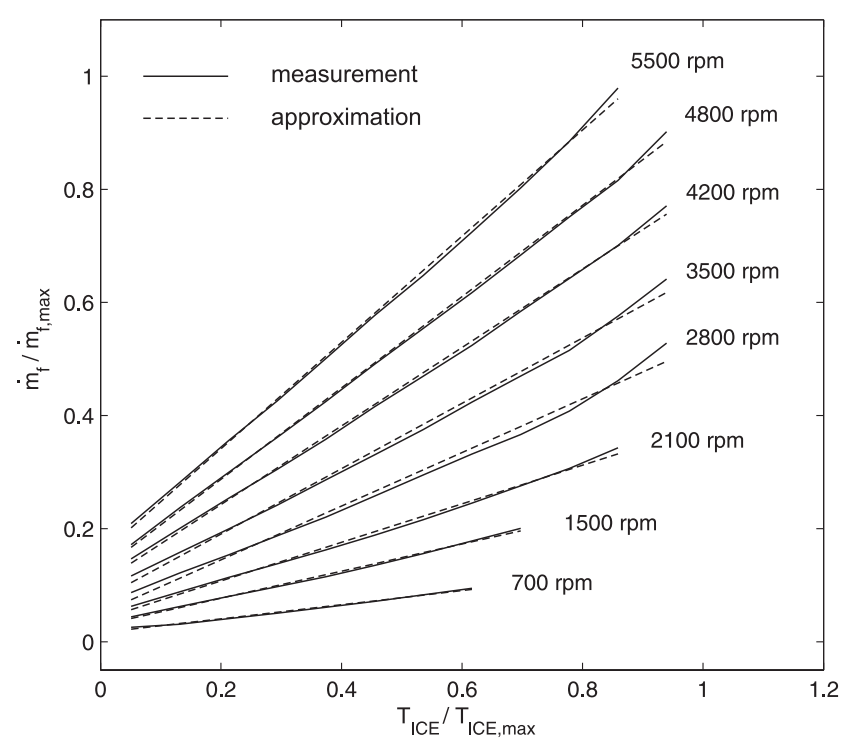

Figure 2

Approximation of the fuel map. 


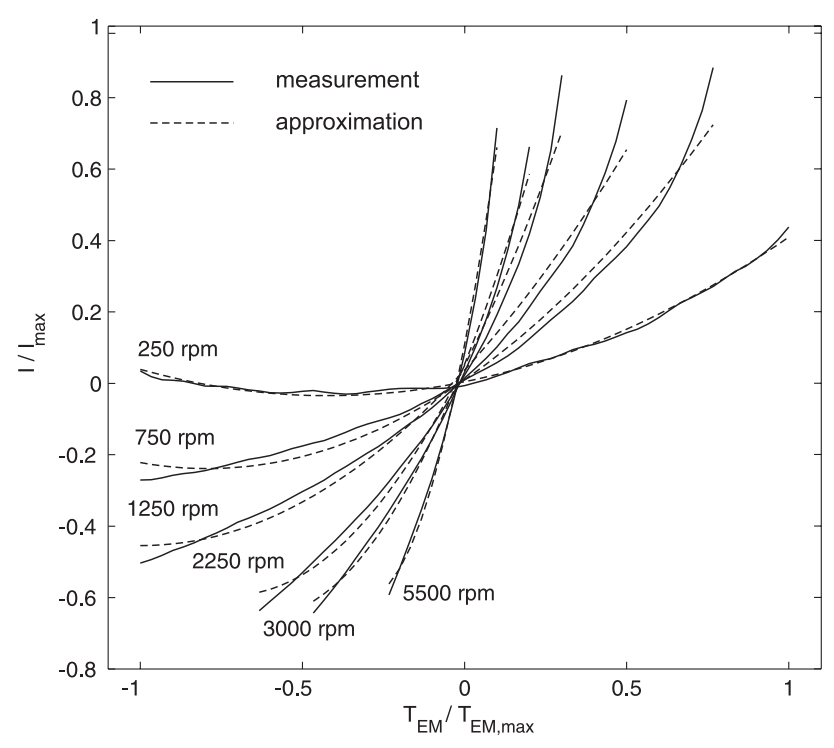

Figure 3

Approximation of the battery current.

is negligible. Furthermore the losses encountered in the electric circuit are roughly quadratic in $T_{E M}$ at a constant angular speed leading to

$$
\dot{\xi} \approx b_{0}\left(\omega_{i n}\right)+b_{1}\left(\omega_{i n}\right) T_{E M}+b_{2}\left(\omega_{i n}\right) T_{E M}^{2}
$$

The quality of this approximation is shown in Figure 3 for the electrical components used for the simulation studies.

Equations (5), (10)-(12) now describe a simple dynamic system which depends quadratically on the input $T_{E M}$ (or $T_{I C E}$ alternatively). $T_{\text {in }}$ and $\omega_{\text {in }}$ are known system parameters that can be directly calculated from $v$ and $\alpha$.

\section{OPTIMAL ENERGY MANAGEMENT}

With the assumption that at the current time $k$ a prediction $v(\cdot \mid k)=(v(k \mid k) \ldots v(k+N \mid k))^{T}$ of the future vehicle speed together with a prediction $\alpha(\cdot \mid k)$ of the road slope is available for the next $N$ time steps, the aim for a fuel efficient power split for the predicted driving conditions can be formulated as

$$
\begin{aligned}
& T_{E M}^{o p t}(\cdot \mid k)=\underset{T_{E M}(\cdot \mid k)}{\operatorname{argmin}} \sum_{i=0}^{N} \Delta T_{s} \dot{m}_{f}(k+i \mid k) \\
& \text { s.t. (1)-(9) } \\
& T_{E M, \min }(\cdot \mid k) \leq T_{E M}(\cdot \mid k) \leq T_{E M, \text { max }}(\cdot \mid k) \\
& \xi(k+N+1 \mid k)=\xi(k)+\sum_{i=0}^{N} \Delta T_{s} \dot{\xi}(k+i \mid k) \geq \xi_{0}
\end{aligned}
$$

with the sampling interval $\Delta T_{s}$ and the reference SOC $\xi_{0}$. The minimum and maximum permissible torques of the electric machine (14) can be calculated observing the ICE, EM and battery limits. Inequality (15) ensures the charge sustaining operation of the HEV. Although $\xi$ is allowed to vary throughout the operation of the vehicle, (15) guarantees that the final SOC at the end of the prediction horizon, which is possibly the end of the complete drive cycle, is at least $\xi_{0}$, whereby $\xi_{0}$ is an optimal SOC around which the battery shall be operated. Usually (15) will be an active constraint at the optimal solution. Only if pure electric propulsion within the horizon is possible, e.g. due to long downhill parts or a high initial $\xi(k), T_{E M}^{o p t}(\cdot \mid k)$ will not be affected by (15). Additional SOC constraints $\xi_{\min }$ and $\xi_{\max }$ which of course have to be observed at every time instant are omitted here for simplicity, since they can be fulfilled implicitly through the choice of the horizon length $N$.

Only the first element of the calculated optimal trajectory $T_{E M}^{o p t}(\cdot \mid k)$ is applied to the drivetrain and the optimisation is repeated in the next sampling instant with updated predictions and an updated system state $\xi(k)$ yielding a receding horizon control strategy.

It is worth noticing that the optimisation variables $T_{E M}(\cdot \mid k)$ are merely coupled through inequality (15), since a certain torque $T_{E M}(k+i \mid k)$ at time $k+i$ neither has an impact on the fuel consumption nor on the torque constraint (14) at any other time instant.

\subsection{Adaptive Equivalence Strategy}

For the sake of simplicity in the following $().(k+i \mid k)$ will be abbreviated by $(.)_{i}$. The property of the optimisation problem mentioned above can be exploited by reformulating (13)-(15) to

$$
\begin{aligned}
& T_{E M}^{o p t}(\cdot \mid k)=\underset{T_{E M}(\cdot \mid k)}{\operatorname{argmin}} \sum_{i=0}^{N} \Delta T_{s}\left(\gamma \dot{m}_{f, i}-\dot{\xi}_{i}\right) \\
& \text { s.t. (1)-(9), (14) }
\end{aligned}
$$

Thereby all optimisation variables are decoupled and the compliance of (15) is left to a correctly chosen equivalence factor $\gamma>0$ which weights the consumption of fuel energy against the usage of electrical energy. Therefore only the local minimisation

$$
\begin{aligned}
& T_{E M}^{o p t}(k \mid k)=\underset{T_{E M}(k \mid k)}{\operatorname{argmin}}\left(\gamma \dot{m}_{f}(k \mid k)-\dot{\xi}(k \mid k)\right) \\
& \text { s.t. (1)-(9) } \\
& T_{E M, \min }(k \mid k) \leq T_{E M}(k \mid k) \leq T_{E M, \text { max }}(k \mid k)
\end{aligned}
$$

has to be performed which decreases the computational burden significantly.

It has been shown in [4] that the choice of a correct constant $\gamma^{o p t}$ leads to the global optimal solution observing charge sustainment. Unfortunately $\gamma^{o p t}$ depends on the actual drive cycle which is not completely known in advance. In addition the control law obtained with (17), (18) 
is very sensitive to $\gamma^{\text {opt }}$. Thus it seems appropriate to choose a variable equivalence factor $\gamma(k)$ that is evaluated online based on past or predicted driving conditions, thereby introducing feedback into the control law $[3,5]$.

The online calculation of $\gamma$ can still be computionally demanding if the nonlinear system description is used, a problem that can be solved by updating the equivalence factor at a slower rate than $T_{E M}^{o p t}(k \mid k)$ [5]. Using the approximations (10)-(12) and the assumption of a constant $\gamma$ throughout the horizon $N$, the optimal input trajectory $T_{E M}^{o p t}(\cdot \mid k)$ can easily be calculated solving a mixed integer quadratic program with only one continuous optimisation variable $T_{E M, i}^{o p t}$ and one decision variable $s_{I C E, i}^{o p t}$ for each time instant $k+i$. Ignoring the torque constraints imposed by (18) the minimum of the quadratic cost function $J$ defined by (17) at $k+i$ is found at

$$
T_{E M, i}^{o p t}= \begin{cases}-\frac{1}{2 b_{2, i}}\left(\gamma a_{1, i}+b_{1, i}\right), & s_{i}=1 \\ T_{i n, i}, & s_{i}=0\end{cases}
$$

leading to an optimal cost function

$$
J_{i}^{o p t}=\left\{\begin{array}{l}
\frac{b_{1, i}^{2}}{4 b_{2, i}}+\gamma\left(a_{0, i}+\frac{a_{1, i} b_{1, i}}{2 b_{2, i}}\right)+\gamma^{2} \frac{a_{1, i}^{2}}{4 b_{2, i}}, \quad s_{i}=1 \\
-b_{1, i} T_{i n, i}-b_{2, i} T_{i n, i}^{2}-\gamma a_{1, i} T_{i n, i}, \quad s_{i}=0
\end{array}\right.
$$

depending on $s_{i}$. The optimum is then given by the torque

$$
T_{E M, i}^{o p t}=\operatorname{argmin}\left(\left.J_{i}^{o p t}\right|_{s_{i}=0},\left.J_{i}^{o p t}\right|_{s_{i}=1}\right)
$$

From (19) and (21) it follows that $T_{E M, i}^{o p t}$ is piecewise linear but discontinuous in $\gamma$ if the quadratic equation $\left.J_{i}^{o p t}\right|_{s_{i}=0}=\left.J_{i}^{o p t}\right|_{s_{i}=1}$, that determines the points of discontinuity, has positive real roots. Additionally there exist a minimum and a maximum $\gamma$ for each time step $k+i$ which limit $\left.T_{E M, i}^{o p t}\right|_{s_{i}=1}$ to the torque constraints (18). From these considerations and approximation (12) it can be concluded that, as the torque $T_{E M, i}^{o p t}$ at each instant $k+i$ is a discontinuous piecewise linear function of $\gamma$, the SOC at the end of the prediction horizon $\xi_{N+1}$ is a discontinuous piecewise quadratic function of $\gamma$. The switching points of this function can be easily assessed based on the above considerations, a detailed description is omitted though. The optimal current equivalence factor $\gamma^{o p t}(k)$ can then be calculated by

$$
\begin{aligned}
& \gamma^{o p t}(k)=\underset{\gamma(k)}{\operatorname{argmax}} \gamma \\
& \text { s.t. } \\
& \gamma>0 \\
& \xi_{N+1} \geq \xi_{0}
\end{aligned}
$$

which can efficiently be done due to the quadratic dependance of $\xi_{N+1}$ on $\gamma$. If the maximisation problem (22)-(24) is not feasible, no charge sustaining operation is possible. $T_{E M}^{o p t}(k \mid k)$ should then be $T_{E M, \min }(k \mid k)$. If, on the other hand, pure electric driving observing charge sustainment is possible, $\frac{1}{\gamma^{o p t}(k)}=0$ and $T_{E M}^{o p t}(k \mid k)=T_{E M, \max }(k \mid k)$. Otherwise $T_{E M}^{o p t}(k \mid k)$ can be calculated from (18)-(21) using $\gamma^{o p t}(k)$.

\subsection{MI-QCLP Strategy}

The approximation of the nonlinear system dynamics offers the opportunity to directly solve the minimisation problem (13)-(15) without using the modification introduced by the equivalence factor. Inserting (10)-(12) and (5) leads to

$$
\begin{aligned}
& {\left[\begin{array}{c}
T_{E M}^{o p t}(\cdot \mid k) \\
s_{I C E}^{o p t}(\cdot \mid k)
\end{array}\right]=\underset{\substack{T_{E M}(\cdot \mid k) \\
s_{I C E}(\cdot k)}}{\operatorname{argmin}}\left(f^{T} T_{E M}(\cdot \mid k)\right.} \\
& \text { s.t. }
\end{aligned}
$$

with

$$
\begin{aligned}
f & =-\Delta T_{s}\left[a_{1}\left(\omega_{i n, 0}\right) \ldots a_{1}\left(\omega_{i n, N}\right)\right]^{T} \\
g & =\Delta T_{s}\left[a_{0}\left(\omega_{i n, 0}\right) \ldots a_{0}\left(\omega_{i n, N}\right)\right]^{T} \\
A & =\Delta T_{s} \operatorname{diag}\left(b_{2}\left(\omega_{i n, 0}\right) \ldots b_{2}\left(\omega_{i n, N}\right)\right) \\
b & =\Delta T_{s}\left[b_{1}\left(\omega_{i n, 0}\right) \ldots b_{1}\left(\omega_{i n, N}\right)\right]^{T} \\
\Delta \xi_{\text {des }} & =\xi_{0}-\xi(k)-1^{T} c \\
c & =\Delta T_{s}\left[b_{0}\left(\omega_{i n, 0}\right) \ldots b_{0}\left(\omega_{i n, N}\right)\right]^{T}
\end{aligned}
$$

This mixed integer quadratically constrained linear program (MI-QCLP) can be solved efficiently due to the specific problem structure. For a fixed $s_{I C E}(\cdot \mid k)$ the solution of the resulting QCLP-subproblem is calculated using an active set strategy. First it must be checked whether (28) constrains the optimal solution. If pure electric driving does not violate (28), the optimal solution is clearly $T_{E M}^{o p t}(\cdot \mid k)=T_{E M, \max }(\cdot \mid k)$. Otherwise the optimum lies on the constraint imposed by (28). In this case the solution is given by

$$
T_{E M}^{o p t}(\cdot \mid k)= \begin{cases}\frac{1}{2} A_{S_{\text {unc }}}^{-1}\left(\lambda f_{S_{\text {unc }}}-b_{S_{\text {unc }}}\right), & T_{E M, i}^{o p t} \in S_{\text {unc }} \\ T_{E M, \text { con }, S_{\text {con }}}(\cdot \mid k), & T_{E M, i}^{o p t} \in S_{c o n}\end{cases}
$$

with the inverse lagrange multiplier

$$
\lambda=+\sqrt{\frac{4 \Delta \xi_{\text {des }, S_{\text {unc }}}+b_{S_{\text {unc }}}^{T} A_{S_{\text {unc }}}^{-1} b_{S_{\text {unc }}}}{f_{S_{\text {unc }}}^{T} A_{S_{u n c}}^{-1} f_{S_{\text {unc }}}}}
$$

The optimal sets $S_{c o n}$ of optimisation variables $T_{E M, i}^{o p t}$ which are constrained by (26) and (27) and $S_{u n c}$ of the 
remaining free optimisation variables can be found starting with $S_{c o n}$ only containing those variables $T_{E M, i}^{o p t}$ fixed by (27) due to $s_{I C E, i}=0$ and then successively calculating the optimal solution according to (35) and (36) and shifting the variables $T_{E M, i}^{o p t}$ that violate (26) from $S_{u n c}$ to $S_{c o n}$ until $T_{E M}^{o p t}(\cdot \mid k)$ is feasible. The expressions (.) $)_{S_{\text {unc }}}$ refer to the matrices and vectors (.) where the entries corresponding to the optimisation variables not contained in $S_{\text {unc }}$ have been removed. Consequently $\Delta \xi_{\text {des }, S_{\text {unc }}}$ is calculated by

$$
\Delta \xi_{d e s, S_{u n c}}=\xi_{0}-\xi(k)-1^{T} c_{S_{u n c}}
$$

$T_{E M, c o n, i}$ is given by $T_{E M, m i n, i}, T_{E M, \max , i}$ or $T_{i n, i}$ depending on the respective active constraint (Equations 26, 27).

If $4 \Delta \xi_{\text {des }, S_{\text {unc }}}+b_{S_{\text {unc }}}^{T} A_{S_{\text {unc }}}^{-1} b_{S_{\text {unc }}}<0$ for a given $s_{I C E}(\cdot \mid k)$, the $\lambda$ calculated by (36) is complex ${ }^{(1)}$. In this case no charge sustaining solution can be found and the optimal solution is

$$
T_{E M}^{o p t}(\cdot \mid k)=T_{E M, \min }(\cdot \mid k), \quad T_{E M, i}^{o p t} \in S_{\text {unc }}
$$

The outer mixed integer optimisation problem of finding the optimal $s_{\text {ICE }}^{\text {opt }}(\cdot \mid k)$ can be solved quickly by modifying $s_{I C E}(\cdot \mid k)$ in a way that eliminates primarily low ICE torque requests resulting from $T_{E M}^{o p t}(\cdot \mid k)$ for a given $s_{I C E}(\cdot \mid k)$.

\subsection{Correlation of the Two Strategies}

The two strategies are closely related to each other. Given an optimal solution $s_{I C E}^{o p t}(\cdot \mid k)$ of the outer mixed integer optimisation and omitting the torque constraints at this point for

(1) A complex-valued $\lambda$ indicates that the optimisation problem (25)-(28) has no feasible solution for the chosen $s_{I C E}(\cdot \mid k)$.
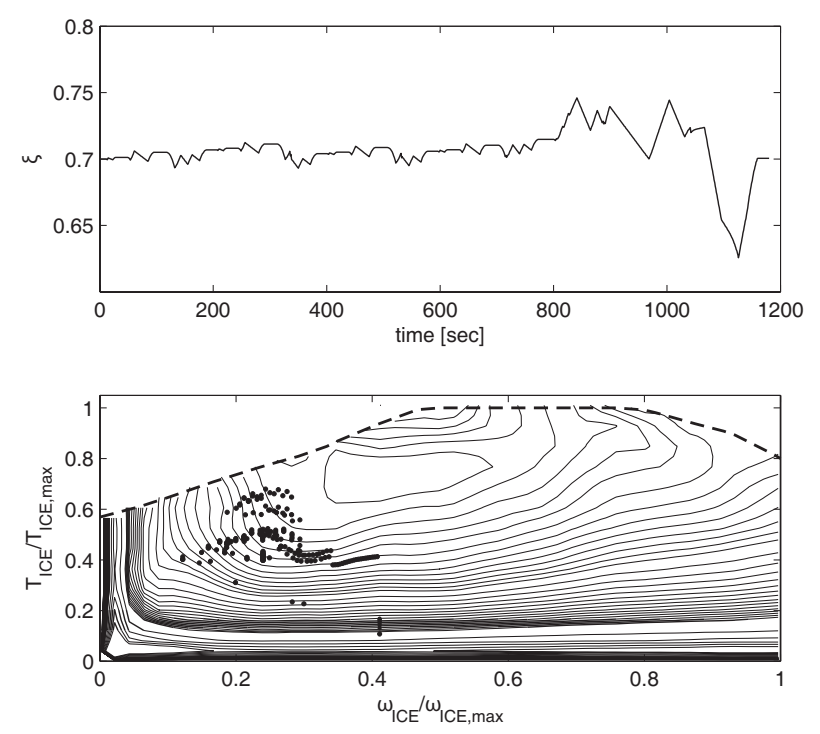

Figure 4

Performance of the Adaptive Equivalence Strategy for the NEDC. simplicity, the resulting optimisation problem for the MIQCLP algorithm is given by

$$
\begin{aligned}
& T_{E M}^{o p t}(\cdot \mid k)=\underset{T_{E M}(\cdot \mid k)}{\operatorname{argmin}} \sum_{i=0}^{N} \Delta T_{s} \dot{m}_{f, i} \\
& \text { s.t. } \\
& \sum_{i=0}^{N} \Delta T_{s} \dot{\xi}_{i}-\Delta \xi_{\text {des }} \geq 0
\end{aligned}
$$

The corresponding Lagrangian

$$
L=\sum_{i=0}^{N} \Delta T_{s}\left(\dot{m}_{f, i}-\mu \dot{\xi}_{i}\right)+\mu \Delta \xi_{d e s}
$$

is minimised by $T_{E M}^{o p t}(\cdot \mid k)$ and the corresponding lagrange multiplier $\mu^{o p t}$ given by $\lambda^{-1}$ according to (36). It is obvious that minimising (41) with respect to $T_{E M}(\cdot \mid k)$ is equivalent to minimising (16) for a constant $\gamma=\mu^{-1}$. Since $\gamma^{o p t}(k)$ is also derived from inequality (40), for the same fixed $s_{I C E}^{o p t}(\cdot \mid k)$ both algorithms yield the same result and

$$
\gamma^{o p t}(k)=\lambda
$$

holds. The different results obtained with both strategies therefore originate from a different solution of the outer mixed integer optimisation problem. While the Adaptive Equivalence approach bases its decision $s_{I C E, i}^{o p t}$ on the value of the Lagrangian at the specific time step $k+i$ (Equations 20,21) without considering other time steps within the prediction horizon, the MI-QCLP algorithm finds $s_{I C E}^{\text {opt }}(\cdot \mid k)$
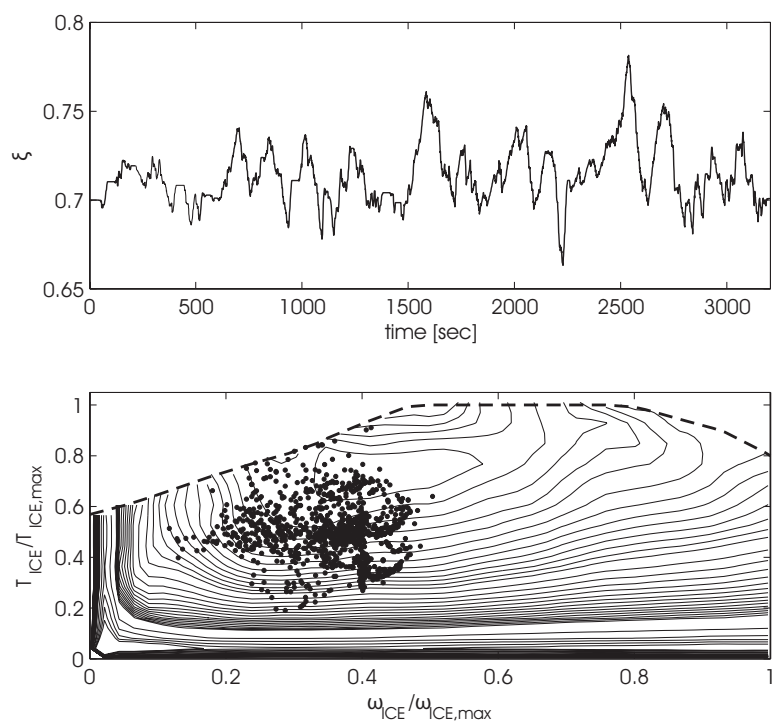

Figure 5

Performance of the Adaptive Equivalence Strategy for the HYZEM cycle. 
on behalf of global considerations. This leads to the expectation, that the MI-QCLP strategy outperforms the Adaptive Equivalence strategy.

\section{SIMULATION RESULTS}

The components chosen for the simulation study are a 5speed automatic gearbox, a conventional gasoline engine, a strong permanent magnet synchronous electric machine which allows pure electric driving and a NiMH-battery pack. In order to check the performance of the developed algorithms, perfect predictive information regarding the vehicle speed and the road slope was assumed. The algorithms were tested in the New European Drive Cycle (NEDC) and the more realistic HYZEM cycle with a sampling rate of $1 \mathrm{sec}$ using (1)-(9) as the nonlinear vehicle model. The simulations were conducted using MATLAB/Simulink. The SOC at the beginning of the cycle and the reference SOC were chosen to $\xi_{\text {start }}=\xi_{0}=0.7$.

\subsection{Adaptive Equivalence Strategy}

Figures 4 and 5 show the results obtained for the NEDC and the HYZEM cycle with the Adaptive Equivalence strategy using a prediction horizon of $N=100$. The final SOC's are $\xi_{\text {end }, N E D C}=0.7005$ and $\xi_{\text {end }, H Y Z E M}=0.7007$ respectively which indicates that the adaptive equivalence factor $\gamma$ is well-determined. The operating points of the ICE exhibit the good performance of the energy management strategy since the low-efficiency regions at low engine loads are omitted.

\subsection{MI-QCLP Strategy}

Figures 6 and 7 show the results for the two drive cycles obtained with the MI-QCLP strategy using a prediction horizon of $N=100$. Although the optimal SOC-trajectories differ from the results calculated with the Adaptive Equivalence strategy, the general behaviour is similar. The ICE only operates at high efficiencies and the operation is charge sustaining $\left(\xi_{\text {end,NEDC }}=0.6951\right.$ and $\xi_{\text {end }, \text { HYZEM }}=0.6996$ respectively).

\subsection{Optimality of the Real-Time Strategies}

In order to assess the optimality of the developed algorithms, the results obtained must be compared to the global optimal solution, which can be calculated offline for the nonlinear system model and a given drive cycle (1)-(9) using the Dynamic Programming (DP) technique.

Figures 8 and 9 compare the global minimal fuel consumption for the NEDC and the HYZEM cycle with the fuel consumptions realised using the two real-time strategies for varying prediction horizons. It can be concluded that the developed algorithms work very well despite the approximations used, whereby the MI-QCLP algorithm yields better results than the Adaptive Equivalence strategy. It should be expected that the obtained fuel consumptions decrease with an increasing prediction horizon since the exploitation of additional information should improve the result. This is indeed the case for the NEDC, nevertheless Figure 9 indicates that the choice of very large horizons deteriorates the results. This is probably caused by the integrating correlation between $\xi_{N+1}$ and approximation (12) which leads to an
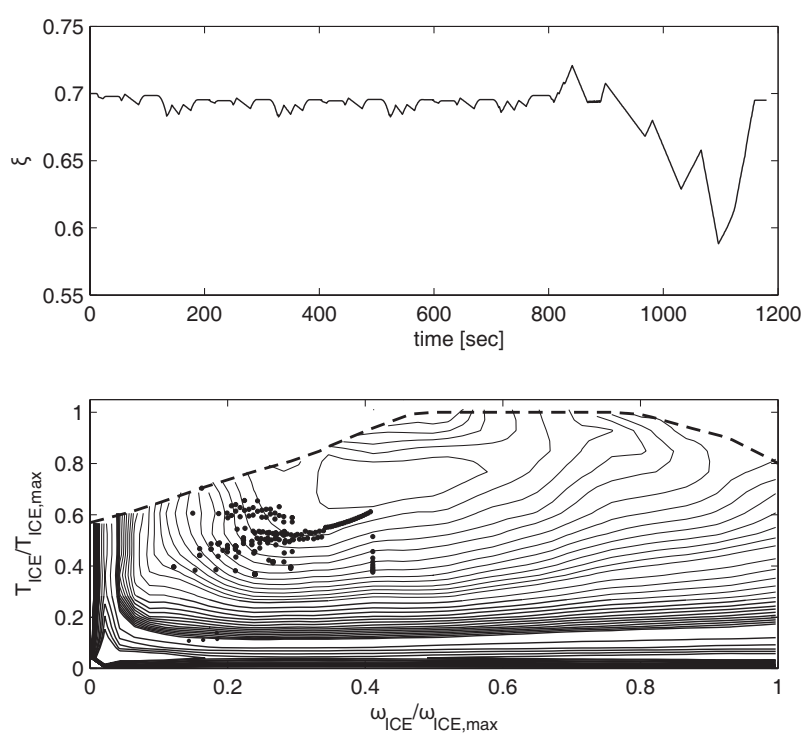

Figure 6

Performance of the MI-QCLP Strategy for the NEDC.
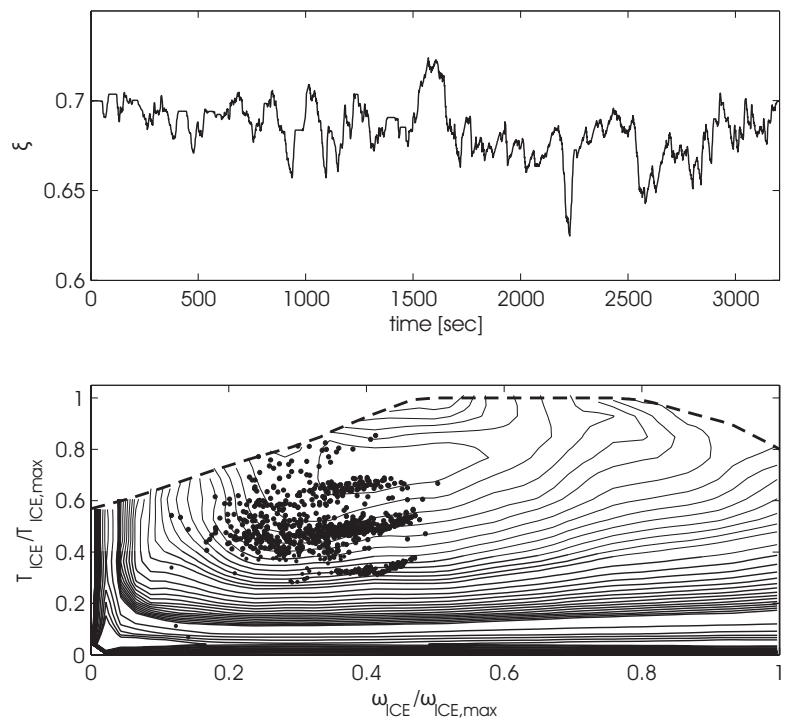

Figure 7

Performance of the MI-QCLP Strategy for the HYZEM cycle. 


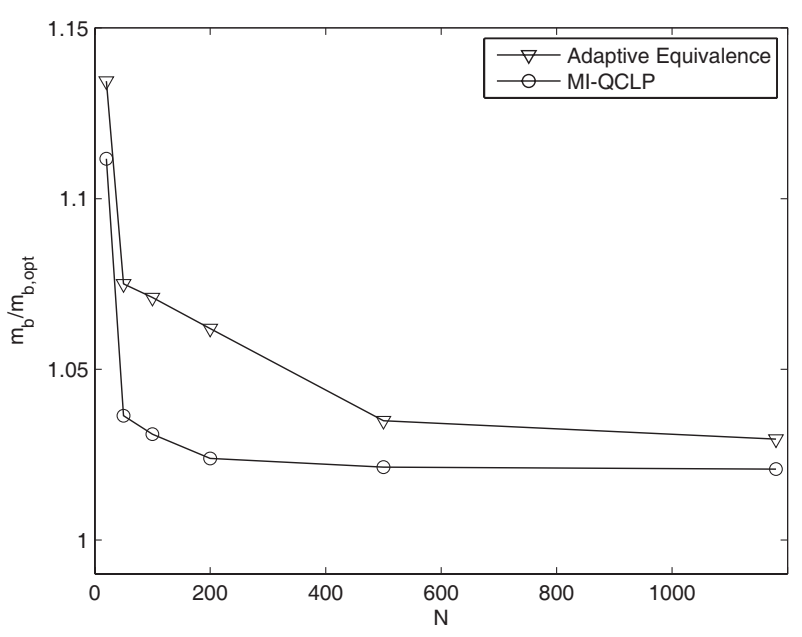

Figure 8

Fuel consumption of the Real-time strategies compared to the global minimum for the NEDC.

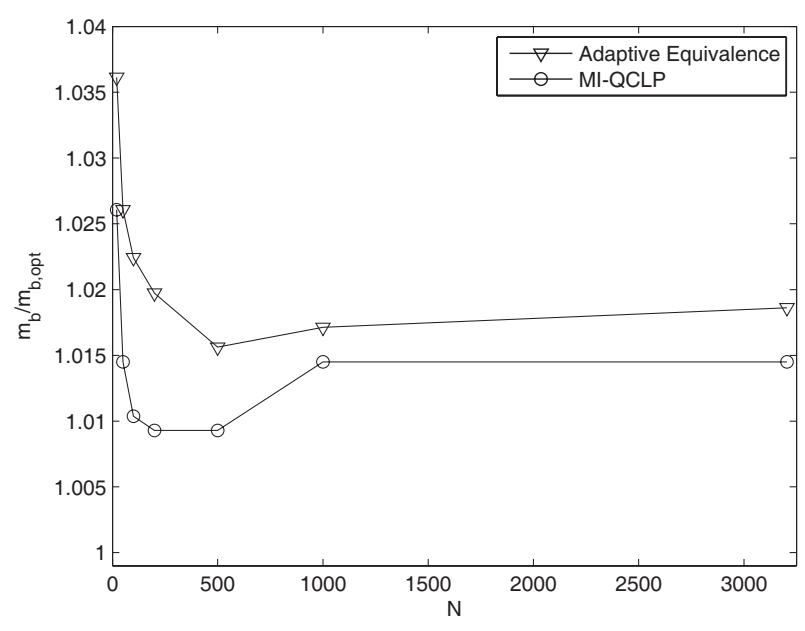

Figure 9

Fuel consumption of the Real-time strategies compared to the global minimum for the HYZEM cycle. increased approximation error for $\xi_{N+1}$ for large horizons $N$. Horizons of $N=100 \ldots 200$ seem a reasonable choice for a good performance. With $N=100$ the MI-QCLP strategy yields a fuel consumption that is within $3.1 \%$ of the global
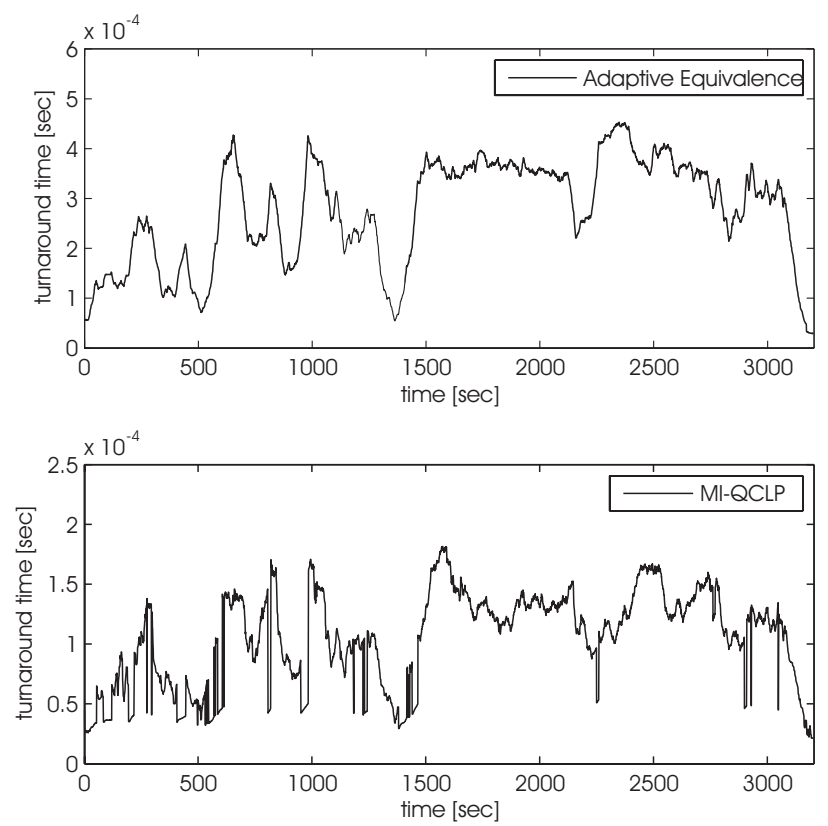

Figure 10

Turnaround times of the Real-time strategies for the HYZEM cycle. minimum for the NEDC and within $1.1 \%$ for the HYZEM cycle.

\subsection{Real-time Capability}

Both strategies have been implemented and tested with a horizon of $N=100$ for the more realistic HYZEM cycle on a $2.6 \mathrm{GHz}$ dspace RCP real-time hardware. The turnaround times needed for the calculation of the optimal solution at a given time instant are displayed in Figure 10. The maximum turnaround time for the MI-QCLP algorithm is $182 \mu \mathrm{s}$. For the Adaptive Equivalence strategy $453 \mu$ s are achieved. These results indicate that the MI-QCLP algorithm is nearly 2.5 times faster than the Adaptive Equivalence strategy which results from the more time-consuming search for the optimal $\gamma(k)$ according to (22)-(24). Nevertheless, considering a sampling time of $10 \mathrm{msec}$ for the energy management controller, both algorithms are real-time applicable even on significantly more limited controller hardware.

\section{CONCLUSION}

This paper presented two different strategies for the optimal energy management of a HEV. Both algorithms incorporate information about future drive conditions for a limited time horizon. Emphasis has been placed on the real-time capability of the operation strategies which allows their implementation in a vehicle. This property could be achieved for both algorithms using an appropriate approximate model of the drivetrain together with adequate objective functions which 
have led to optimisation problems that can be efficiently solved.

Both strategies have been tested in two different drive cycles and the achieved fuel consumptions were compared to the global minimum found by a Dynamic Program. It could be demonstrated that both strategies achieve very good results that lie within a few percent of the true optimum despite the approximate model. It could also be concluded that a direct minimisation of the fuel consumption observing the charge sustainment constraint explicitly outperforms the strategy which relies on an appropriate weighting of fuel and electric energy both regarding the achieved fuel consumptions and the computational effort.

Up to this point the simulations have been carried out using perfect predictive information which obviously can not be provided in reality. Therefore future investigations will focus on the deterioration of the results obtained by the energy management algorithms through imprecise predictive information. Due to the receding horizon strategy and the averaging nature of (15) a certain robustness can be expected which has to be validated through further simulations and real drive tests.

\section{REFERENCES}

1 Back, M. and Terwen, S. (2003) Predictive Control with Dynamic Programming for First Order Nonlinear Systems. Automatisierungstechnik, 51, 547-554.

2 Back, M., Simons, M., Kirschbaum, F. and Krebs, V. (2002) Predictive Control of Drivetrains. Proc. of the 15th IFAC World Congress, Barcelona, Spain, July 21-26, 2002.

3 Sciaretta, A., Back, M. and Guzzella, L. (2004) Optimal Control of Parallel Hybrid Electric Vehicles. IEEE Trans. Contr. Syst. T., 12, 352-363.

4 Sciaretta, A., Guzzella, L. and Onder, C.H. (2003) On the Power Split Control of Parallel Hybrid Vehicles: from Global Optimization towards Real-time Control. Automatisierungstechnik, 51, 195-203.

5 Musardo, C., Rizzoni, G., Guezennec, B. and Staccia, B. (2005) A-ECMS: An Adaptive Algorithm for Hybrid Electric Vehicle Energy Management. Eur. J. Control, 11, 509-524.

6 Rizzoni, G., Guzzella, L. and Baumann, B.M. (1999) Unified Modeling of Hybrid Electric Vehicle Drivetrains. IEEE/ASME Trans. Mechatron., 4, 246-257.

Final manuscript received in October 2006 